\title{
Inflation and Rapid Expansion in a Variable G Model
}

\author{
Christopher Pilot \\ Professor of Physics, Retired, Maine Maritime Academy, Castine, USA \\ Email:chris.pilot43@gmail.com
}

How to cite this paper: Pilot, C. (2020) Inflation and Rapid Expansion in a Variable G Model. International Journal of Astronomy and Astrophysics, 10, 334-345. https://doi.org/10.4236/ijaa.2020.104018

Received: October 13, 2020

Accepted: December 8, 2020

Published: December 11, 2020

Copyright $\odot 2020$ by author(s) and Scientific Research Publishing Inc. This work is licensed under the Creative Commons Attribution International License (CC BY 4.0).

http://creativecommons.org/licenses/by/4.0/

\begin{abstract}
Cosmic inflation is considered assuming a cosmologically varying Newtonian gravitational constant, $G$. Utilizing two specific models for, $G^{-1}(a)$, where, $a$, is the cosmic scale parameter, we find that the Hubble parameter, $H$, at inception of $G^{-1}$, may be as high as $7.56 \mathrm{E} 53 \mathrm{~km} /(\mathrm{s} \mathrm{Mpc})$ for model A, or, 8.55 $\mathrm{E} 53 \mathrm{~km} /(\mathrm{s} \mathrm{Mpc})$ for model B, making these good candidates for inflation. The Hubble parameter is inextricably linked to $G$ by Friedmanns' equation, and if $G$ did not exist prior to an inception temperature, then neither did expansion. The CBR temperatures at inception of $G^{-1}$ are estimated to equal, 6.20 E21 Kelvin for model A, and 7.01 E21 for model B, somewhat lower than CBR temperatures usually associated with inflation. These temperatures would fix the size of Lemaitre universe in the vicinity of $3 \%$ of the Earths' radius at the beginning of expansion, thus avoiding a singularity, as is the case in the $\Lambda \mathrm{CDM}$ model. In the later universe, a variable $G$ model cannot be dismissed based on SNIa events. In fact, there is now some compelling astronomical evidence, using rise times and luminosity, which we discuss, where it could be argued that SNIa events can only be used as good standard candles if a variation in $G$ is taken into account. Dark energy may have more to do with a weakening $\mathrm{G}$ with increasing cosmological time, versus an unanticipated acceleration of the universe, in the late stage of cosmic evolution.
\end{abstract}

\section{Keywords}

Cosmic Inflation, Variable G Model, SNIa Events, Dark Energy, Lemaitre Cosmology

\section{Introduction, Cosmic Inflation with a Varying G}

The Friedman equations and the $\Lambda \mathrm{CDM}$ model both assume that Newton's constant, $G$, is a true constant of nature. Recently [1] [2], it was argued that this may 
not be the case. Actually, a variable $G$ has a long history going back to the early works of Dirac in his large number hypothesis (LNH) [3] [4] [5], and Jordan [6] [7] [8] [9]. They both claimed that $G$ must vary as a function of cosmological time, and moreover, in the case of Jordan, that $G$ must be related to Hubble's parameter via the relation, $\dot{G} / G=-H$. Jordan also introduced [6] a scalar field, $\varphi$, already in 1937, within a year of Dirac's LNH, to represent Newton's constant, realizing that $G$ is now some sort of order parameter. The history of a variable $G$ is long and extensive, and will not be repeated here. There have been very many theoretical and observational attempts to measure a variation in $G$, if it indeed exists. Some of those attempts have been presented in reference [1], and we refer the reader to that work, and references therein.

In reference [1], we sought an explanation for the cosmological constant problem. We assumed that the quintessence parameter, $w$, is not precisely equal to, -1 , as in the $\Lambda \mathrm{CDM}$ model but rather, that its value is closer to, $w=-0.98$, as measured observationally. Within observational error, however, $w=-1$, can easily be accommodated, but perhaps this is not its exact value. Assuming that, $w=-0.98$, we were able to demonstrate that, $\dot{G} / G=-0.06 H$, in the current epoch, a value within present observational bounds. The dot over a quantity represents a derivative with respect to cosmological time. Jordan's original hypothesis that, $\dot{G} / G=-H$, seems to be ruled out by observational evidence, but not, $\dot{G} / G=-0.06 H$. This represents a very slight variation, in the present epoch, but in previous epochs, $\dot{G} / G$, or, $G^{-1} \mathrm{~d} G(a) / \mathrm{d} a$, where "a" equals the cosmic scale parameter, was much more drastic. Our theory and models went further and gave a new cosmology assuming that $G$ does vary. In the limit where, $w \rightarrow-1$, we retrieve all the standard results of the $\Lambda \mathrm{CDM}$ model. Our two models deviate appreciably from the $\Lambda$ CDM model only at relatively high CBR temperatures. In this short paper, we wish to discuss some of the implications for inflation. We also wish to consider some of the ramifications in the more recent epochs, where dark energy dominates.

Inflation is a theory [10] [11] [12] [13], which is needed to explain the homogeneity, as well as the perturbations, associated the CBR temperature maps, obtained from WMAP/Planck satellite data. What is mapped occurred at photon matter decoupling, the era of last scattering, roughly 380,000 years after the Big Bang. A so-called "inflaton" field is assumed much earlier, which causes a rapid and drastic, almost explosive, expansion of the universe in its very earliest development, within 10E-32 seconds after the big bang. Within this fraction of a second, the entire universe went from roughly the size of a proton to the size of a ball, roughly $10 \mathrm{~cm}$ across [10]. Not all physicists are comfortable with this idea. A-causal expansion is required where the Hubble envelop expands at faster than the speed of light. It is also a mind-boggling thought trying to imagine such a physical process, where the entire universe, as we know it, can be collapsed to what is, essentially, a singularity.

In this paper, we argue for a different interpretation, one incorporating a vari- 
ation in $G$ with respect to cosmological time. In reference [1], we introduced two specific models for, $G^{-1}$, which we called models, A, and, B. Both were one parameter, non-linear functions, which mimic order parameter behavior. We believe that $G^{-1}$ is an intrinsic property of the vacuum, which involves some sort of self-organization within the vacuum, i.e., space. It is purely an artifact of space, which does not necessarily involve ordinary mass, made up of quarks and leptons. In fact, we know that the Planck mass, $M_{P l}$, and $G$ are related by the equation,

$$
M_{P l}=(\hbar c / G)^{1 / 2}
$$

We square this result, and claim moreover, that $G^{-1}$ is an order parameter, satisfying,

$$
M_{P l}^{2}=\hbar c G^{-1}=\left\langle 0\left|\varphi^{2}\right| 0\right\rangle
$$

Here, the, $M_{P l}^{2}$, is no longer a constant, but the vacuum expectation value (VEV) of a scalar field, $\varphi$, squared. As the scalar field, $\varphi$, freezes out of the vacuum, $G^{-1}$ changes its value, a process lasting eons. In our scenario, $G^{-1}$ is no longer a constant, and neither is the Planck mass. We identify the scalar field in Equation (1-2), with the scalar field of Jordan, first introduced in 1937, but largely ignored when discussing inflation.

It should be noted that $M_{P l}^{2}$ has the same canonical dimensions as magnetization in condensed matter physics, or $M_{W_{ \pm}}^{2}$ in particle physics. In the electro-weak interaction, $M_{W_{ \pm}}$, is the mass of the $W_{ \pm}$boson. Thus, it could very well be an order parameter based on inherent dimension alone. It is well known that $M_{W_{+}}^{2}$ is essentially the inverse Fermi constant, $G_{F}^{-1}$, which fades at high energies, and is only constant well below $100 \mathrm{GeV}$. Newton's constant, and the Fermi constant, are the only two known coupling constants in physics, which have an inherent dimension, and that canonical dimension is the same for both. It can be expressed as inverse mass squared.

Model A, assumes [1] a $G^{-1}$ scaling law as follows:

$$
G^{-1}(T)=G_{\infty}^{-1}\left(1-\mathrm{e}^{b / T}\right) \quad(\text { model A) }
$$

In this equation, $T$ is the $C B R$ temperature of the universe and $G_{\infty}^{-1}$ is a saturation value, achieved in the limit where, $T \rightarrow 0$. The constant " $b$ " has units of temperature, but is independent of temperature. In model $\mathrm{A}$, the constant, $b$, equals, $b=11.663$, which was determined by demanding that, $w=-0.98$. Also, $G_{\infty}^{-1}=1.014 G_{0}^{-1}$, where $G_{0}$ is Newton's constant. In the current epoch we have the well-known, $G_{0}=6.674 \mathrm{E}-11$, in MKS units. All units, which are not explicitly written out in this paper, are MKS units. The cosmic scale parameter, " $a$ ", can be expressed as, $a=(1+z)^{-1}=T_{0} / T=R / R_{0}$, where $T$ is the CBR temperature, and in the present epoch, $T_{0}=2.725$ Kelvin . Using this relation, Equation (1-3), can be rewritten as,

$$
G^{-1}(T)=G_{\infty}^{-1}\left(1-\mathrm{e}^{-4.28 a}\right) \quad \text { (model A) }
$$

This equation came into being at a temperature estimated [1] to be, 6.20E21 
Kelvin. We are also close to full saturation in the present epoch since, $G_{\infty}^{-1}=1.014 G_{0}^{-1}$. Saturation for all practical purposes, will occur at 10 times the current Hubble radius in this model, or when, $a \cong 10$. We are using the convention where $a_{0}=1$. Equations (1-3) and (1-4), are modeled as a charging capacitor, and we call model A our charging capacitor model. What is charging up as a function of decreasing CBR temperature is the Planck mass squared, or the VEV, of, $\varphi^{2}$, as seen explicitly in Equation (1-2). As mentioned, this is a very time consuming process, covering over 20 orders of magnitude, CBR temperature wise.

Model B, assumes an entirely different scaling law, namely,

$$
G^{-1}(T)=G_{\infty}^{-1}[\operatorname{coth}(b / T)-T / b] \quad(\text { model B) }
$$

Again, $G_{\infty}^{-1}$ is the saturated value, applicable in the limit where the CBR temperature, $T \rightarrow 0$. We could just as well have called, $G_{\infty}^{-1}=G_{T=0}^{-1}$. The constant, " $b$ ", having units of Kelvin, has been determined to equal, $b=48.15$ Kelvin, in order to guarantee that the quintessence parameter, $w=-0.98$. Again, the parameter, " $b$ ", per se, is independent of CBR temperature, even though it is measured in units of Kelvin. Here, in model B, it is found that, $G_{\infty}^{-1}=1.054 G_{0}^{-1}$.

The nonlinear function, Equation (1-5), is recognized as the Langevin function, defined as, $L(x) \equiv \operatorname{coth}(x)-1 / x$, used in magnetism. This is another approach towards modeling, $G^{-1}(a)$, and we call this model B. The quantity, $G^{-1}(a)$, has inherent canonical dimension of magnetization. Referring to equation (1-2), we treat $\left\langle 0\left|\varphi^{2}\right| 0\right\rangle$ as an order parameter, much like magnetization. In terms of canonical dimension, magnetization has the same units as inverse mass squared, or inverse momentum squared.

Another way to rewrite Equation (1-5), is to remember that, $a=(1+z)^{-1}=T_{0} / T=R / R_{0}$, where $z$ is the redshift, and $R$, the Hubble radius. The temperature, $T_{0}=2.725$, is the current CBR temperature. We substitute " $a$ " in place of temperature, using the above expression for, “a”. In Equation (1-5), this gives,

$$
G^{-1}(a)=G_{\infty}^{-1}[\operatorname{coth}(17.67 a)-1 /(17.67 a)] \quad(\operatorname{model} \mathrm{B})
$$

This order parameter surfaced [1] at a Curie temperature of, 7.01E21 Kelvin, which is very, very close to the value indicated by model A. Even though the two underlying functions, Equations (1-4) and (1-6), are entirely different and distinct functions, they lead, remarkably, to approximately the same inception temperature. The order of magnitude is perfect.

In model B, the current value for Newton's constant, $G_{0}$, is not far from the final saturation value. In fact, we have the relation, $G_{\infty}^{-1}=1.054 G_{0}^{-1}$. Saturation in model B, will effectively occur at a CBR temperature, roughly half the present temperature of, 2.725 Kelvin. The universe will then have expanded its Hubble radius to twice its present value.

When plotted separately as a function of " $a$ ", the scale parameter, both model A, and, model B, functions look very similar. At inception, $G^{-1}$, will rise very 
dramatically from zero. This is because, at very high temperatures, the $G^{-1}$ is proportional to inverse temperature, $T^{-1}$, in both models. As the CBR temperature decreases upon expansion, $G^{-1}$ will start to level off, and eventually start approaching a saturation value, indicated by, $G_{\infty}^{-1}$. Currently, in the present epoch, we are close to saturation and that is the reason why $\dot{G} / G$ is very nearly equal to zero. In the current epoch, $\dot{G} / G$ is of the order, $10 \mathrm{E}-12$ per year, a very small value.

Both models $\mathrm{A}$ and $\mathrm{B}$, indicate inception temperatures of, 6.20E21 Kelvin, and, 7.01E21 Kelvin, respectively. Since $G^{-1}$ did not really exist prior to that point in cosmological time, neither did, $G$. And, in the simplest form of the Friedmann equation,

$$
H^{2}=8 \pi G \rho / 3
$$

this would indicate that the universe was not expanding at all until the temperature dropped down to the inception temperature. What existed beforehand, or what caused the universe to suddenly expand, is unknown. But as to what happened shortly thereafter, we have an idea given these two models for, $G^{-1}$.

It turns out that using Equations (1-3), and (1-5), we can find the values of $G$ at inception. Let $G_{C}=G\left(T_{C}\right)$ where, $T_{C}$ is the Curie temperature. Then using the inception temperatures listed above, we find,

$$
\begin{aligned}
& G_{C} / G_{0}=5.27 \mathrm{E} 20 \quad(\text { model A) } \\
& G_{C} / G_{0}=4.11 \mathrm{E} 20 \quad \text { (model B) }
\end{aligned}
$$

The high $G_{C} / G_{0}$ values, indicated by Equations (1-8a, b), were needed to explain the cosmological constant problem, as shown in reference [1]. The cosmological constant, $\Lambda$, equals, $\Lambda=8 \pi G \rho_{\Lambda} / c^{2}$, where $\rho_{\Lambda}$ is the mass density associated with dark energy. We see that $\Lambda$ is related to both, $G$, and $\rho_{\Lambda}$, and if $G$ had a very large value in prior epochs, this would help explain the gross disparity between present, and early universe, $\Lambda$ values. We can prove, namely, that [1], $\Lambda / \Lambda_{0}=\left(G / G_{0}\right)^{2}$. The, $G_{0}$, refers, as always, to the current value for Newton's constant.

We next wish to find the corresponding Hubble parameter values at inception of, $G^{-1}$. We will use Equation (1-7). The $H_{C}$ values would indicate the rate of expansion at roughly, 10E21 Kelvin. To determine, $H_{C}$, we first need to estimate the mass/energy density, $\rho_{C}$, at these very high temperatures. At temperatures of the order, 10E21 Kelvin, no elementary particles have yet condensed out of the vacuum. All quarks and leptons, i.e., all matter particles in the standard model, only froze out at lessor temperatures, below 10E16 Kelvin or approximately, $1 \mathrm{TeV}$ [14] [15] [16] [17]. So what remained above this temperature? As far as we can tell, only radiation existed, in the form of blackbody photons and, possibly, blackbody neutrinos. The energy density, or equivalent mass density, for blackbody radiation scales as, $a^{-4}$, as is well known. And the current estimate for the equivalent mass density is about, $\Omega_{\text {Radiation }} \rho_{0}=(8.3 \mathrm{E}-5) \rho_{0}$, where, $\rho_{0}=8.624 \mathrm{E}-27 \mathrm{~kg} / \mathrm{m}^{3}$ is the total mass/energy density in the current epoch. 
Therefore the radiative energy density at inception must equal,

$$
\rho_{C}=a_{C}^{-4}(8.3 \mathrm{E}-5)(8.624 \mathrm{E}-27)
$$

This, however, is only part of the story, because we have not taken into account the masses of the known elementary particles. When their flavors, and their degrees of freedom, are taken into account, we should properly multiply Equation (1-9), by a correction factor, $g^{*}=106.75$. See references [16] [17]. This still does not take into account dark matter, nor dark energy, but it does factor in what is definitely known. If we multiply Equation (2-9), by this correction factor, $g^{*}=106.75$, we will have effectively taken into account, as well, the radiation that coalesced as material particles between then and now.

What remains is to find $a_{C}$. But this is easy since we know the temperatures at inception, and, $a_{C}=T_{0} / T_{C}$. For models $\mathrm{A}$, and $\mathrm{B}$, we find, respectively, that

$$
\begin{array}{ll}
a_{C}=T_{0} / T_{C}=4.40 \mathrm{E}-22 & (\text { model A) } \\
a_{C}=T_{0} / T_{C}=3.89 \mathrm{E}-22 & (\text { model } \mathrm{B})
\end{array}
$$

These values are very close to one other because the inception temperatures were nearly equal. We next substitute these cosmic scale factors into Equation (1-9), and multiply the result by the correction factor, $g^{*}=106.75$, to take masses, which were frozen out below $1 \mathrm{TeV}$, into account. The results are, respectively,

$$
\begin{array}{ll}
\rho_{\text {Radiation }, C}=\rho_{\text {Radiation }}\left(T_{C}\right)=2.04 \mathrm{E} 57 & (\text { model A }) \\
\rho_{\text {Radiation }, C}=\rho_{\text {Radiation }}\left(T_{C}\right)=3.34 \mathrm{E} 57 & (\text { model B })
\end{array}
$$

These are the values, which we will substitute into Equation (1-7). Keep in mind that Newton's constant gets replaced by a new value, $G_{C}$, when we do that. Those values are given by Equations (1-8a), and (1-8b), respectively.

We do this next. We substitute Equations (1-11a, b), and (1-8a, b), into Equation (1-7), and find, respectively, that the Hubble parameter equals

$$
\begin{array}{ll}
H_{C}=2.45 \mathrm{E} 34 \text { seconds }^{-1} & (\text { model } \mathrm{A}) \\
H_{C}=2.77 \mathrm{E} 34 \operatorname{seconds}^{-1} & (\text { model B) }
\end{array}
$$

In MKS units, the Hubble parameter is measured in inverse seconds. Converting to the more standard, $\mathrm{km} /(\mathrm{s} \cdot \mathrm{Mpc})$, we obtain,

$$
\begin{array}{ll}
H_{C}=7.56 \mathrm{E} 53 \mathrm{~km} /(\mathrm{s} \cdot \mathrm{Mpc}) & (\text { model A) } \\
H_{C}=8.55 \mathrm{E} 53 \mathrm{~km} /(\mathrm{s} \cdot \mathrm{Mpc}) & (\text { model } \mathrm{B})
\end{array}
$$

Needless to say, these expansion rates are dramatic, especially in light of the fact that the current value of $H_{0}$ equals a mere $67.74 \mathrm{~km} /(\mathrm{s} \cdot \mathrm{Mpc})$. Our view is that these high values indicate inflation. The so-called inflaton field, usually associated with inflation, will be replaced by a scalar field, $\varphi$, first introduced by Jordan in 1937, and highlighted in Equation (1-2). Equations (1-13a) or (1-13b), is our version of inflation.

As mentioned, what happens before this point in cosmological time is any- 
one's guess. How the CBR temperature dropped down to, 10E21 Kelvin, in the first place if the universe didn't expand prior to that point, is also a mystery. Interestingly, when Lemaitre first introduced his expanding universe hypothesis in the mid 1920's, he never claimed that it evolved from a singularity. In fact, early critics derided his hypothesis by referring to it as his "cosmic egg" hypothesis [18] [19] [20] [21]. We are advocating this view however... the universe did not start from a singularity. It had a finite size, and a finite CBR temperature, before it started to expand.

The finite size can be estimated at the start of expansion. Since, $a=R / R_{0}=T_{0} / T$, we can set, $R_{C}=a_{C} R_{0}$. Using a currently accepted estimate [22] [23] for the Hubble radius in the present epoch, $R_{0} \cong 4.4 \mathrm{E} 26$ meters, we find using equations, (1-10a, b), that

$$
\begin{array}{ll}
R_{C}=a_{C} R_{0}=1.94 \mathrm{E} 5 \text { meters } & (\text { model } \mathrm{A}) \\
R_{C}=a_{C} R_{0}=1.71 \mathrm{E} 5 \text { meters } & (\text { model } \mathrm{B})
\end{array}
$$

This is roughly 3 percent of the earth's radius. This would be our initial estimate for the size of Lemaitre's cosmic egg at the beginning of expansion.

\section{Dark Energy and Subsequent Late Epoch Development}

We now turn to the later stages of cosmic evolution where we have dark energy. We want to consider, specifically, the increased luminosity distance associated with SNIa events, which led one to conclude that the universe is currently expanding more rapidly, than anticipated.

Consider a specific SNIa event, where the energy flux, measured on earth bound satellites, fixes a specific distance to the source using the luminosity-distance-flux relation. SNIa events, i.e., explosions, make for good standard candles because of their brightness, and excellent predictable luminosity. The observed distances, measured in the late 1990's, and after, suggest that the universe is expanding faster than we thought. We have an unanticipated acceleration leading one to surmise, erroneously we believe, that in the later stages of cosmic evolution, a type of antigravity or negative pressure surfaces, which we identify as dark energy. What happens however, if $G$ varies cosmologically?

The luminosity of a SNIa event varies as $G^{-3 / 2}$ [24] [25] [26] [27], and if $G$ is unequal to a constant, the luminosity would vary. Since $G^{-1}$ increases as cosmological time increases, $G^{-3 / 2}$ must also increase. So gravity gets weaker as time progresses, and the universe would appear to accelerate more expansion wise. The two functions that we introduced for $G^{-1}$ in reference [1] can be used to model this behavior.

Taking this a step further, we know that the energy flux received by an observer here on earth is related to the luminosity distance by the relation, $\Phi=L /\left(4 \pi d_{L}^{2}\right)$, where $\Phi$ is the flux, $d_{L}$, the luminosity distance, and, $L$, the luminosity. Thus, if $d_{L}$ seems to increase unexpectedly, it may actually be due to $L$ decreasing if one goes back in cosmological time. At higher redshifts, the luminosity, $L$, could be weaker. Let $L_{0}$ be the luminosity in the present epoch, 
and set $d_{L}$ equal to the expected distance without dark energy. Then the observed flux gives,

$$
L_{0} / d_{L}^{\prime 2}=L / d_{L}^{2}=\left(G / G_{0}\right)^{-3 / 2} L_{0} / d_{L}^{2}
$$

In this equation, $d_{L}^{\prime}$ is the perceived and un-anticipated, accelerated luminosity distance, leading to the notion of dark energy. By contrast, $d_{L}$, is the true luminosity distance. The increase in $d_{L}^{\prime}$ over $d_{L}$ is really due to a decrease in $G^{-1}$ if one goes back in time. Equation, (2-1), can namely be rewritten as

$$
d_{L}^{\prime} / d_{L}=\left(G / G_{0}\right)^{3 / 4}
$$

It is apparent by this equation, that if $G$ increases, then, $d_{L}^{\prime}$ also increases. Depending on the look-back time, at a specific redshift, the $G$ is stronger in value, and thus by Equation (2-2), this makes the ratio, $d_{L}^{\prime} / d_{L}$, larger.

Models A, and B, for $G=G(a)=G(z)$, introduced in the last section, can now be substituted for the right hand side of Equation (2-2). We can predict the amount of unanticipated luminosity distance as a function of redshift since, $a=(1+z)^{-1}$. Antigravity, or negative pressure, we argue, is caused by a declining $G$ value as cosmological time advances. Or, if we go back in time, we obtain a larger $d_{L}^{\prime}$ value.

As specific examples of accelerated expansion, we calculate $\left(G / G_{0}\right)$ at a redshift of, $z=1 / 2$, and then again, at, $z=2 / 3$. First, consider, $z=1 / 2$. Since, $a=(1+z)^{-1}$, this corresponds to $a=2 / 3$. Equation, (1-4), model A, gives

$$
G / G_{0}=G_{0}^{-1} / G^{-1}=\left[1-\mathrm{e}^{-4.28}\right] /\left[1-\mathrm{e}^{-(4.28 \times 2 / 3)}\right]=1.0465 \text { (model A) }
$$

Substituting this into Equation (2-2), we obtain

$$
d_{L}^{\prime} / d_{L}=1.035 \quad(z=1 / 2, \text { model } \mathrm{A})
$$

For model B, we use Equation (1-6). Here,

$$
\begin{aligned}
G / G_{0} & =G_{0}^{-1} / G^{-1} \\
& =[\operatorname{coth}(17.67)-1 / 17.67] /[\operatorname{coth}(17.67 \times 2 / 3)-3 /(17.67 \times 2)](\operatorname{model} \text { B) }(2-5) \\
& =1.031
\end{aligned}
$$

And therefore, by Equation (2-2), we expect,

$$
d_{L}^{\prime} / d_{L}=1.023 \quad(z=1 / 2, \text { model B })
$$

We emphasize that both functions, Equation (1-4), and Equation (1-6), for $G^{-1}(a)$, are nonlinear.

Let us now carry out the same analysis for one further redshift. We now consider a redshift, $z=2 / 3$, or, $a=(1+2 / 3)^{-1}=0.6$. Following the same steps as before, but for this new redshift, we obtain for model $\mathrm{A}$,

$$
d_{L}^{\prime} / d_{L}=1.051 \quad(z=2 / 3, \text { model A })
$$

For model B, this ratio becomes,

$$
d_{L}^{\prime} / d_{L}=1.036 \quad(z=2 / 3, \text { model B })
$$

We have a clear increase in luminosity distance in Equations (2-7), and (2-8), 
when they are compared to Equations (2-4), and (2-6), respectively. We also notice that model, $\mathrm{A}$, is more aggressive than model $\mathrm{B}$, in predicting increases in, $d_{L}^{\prime} / d_{L}$, at a specific $z$ value.

There is some astronomical evidence that higher redshifted SNIa luminosities are weaker. Thorsett [24], analyzed the energy release in SNIa explosions both near, at low $z$, and far, at higher $z$. The lookback times were between $1 \mathrm{Gyr}$, and, 12 - 13 Gyr. He obtained $\dot{G} / G=-0.6 \pm 4.2 \mathrm{E}-12 \mathrm{yr}^{-1}$. He took a linear average, and thus, his results cannot be directly compared to our nonlinear functions for, $G^{-1}$. However, his results indicate a clear weakening, within experimental error, in luminosity. In earlier epochs, SNIa events were just not as powerful, as they are today.

Not only is luminosity affected by Newton's constant $G$, but also, $\tau$, the rise and fall time, for SNIa events [25] [26] [27]. The width of the peak of the light curve, which we call, $\tau$, is proportional to $G^{-3 / 4}$. Rise and fall times between, $\tau=17.50 \pm 0.4$ days , and, $\tau=19.95 \pm 0.15$ days, have been observed at high $Z$, and low $Z$, SNIa events, respectively. So not only are the luminosities weaker for high $z$ events, but also, $\tau$, the width of the light curve. In previous epochs, these explosions did not last as long, if this theory is correct.

In summary, a $G^{-3 / 2}$ behavior for luminosity, and a $G^{-3 / 4}$ behavior for light curve widths, cannot be dismissed in a variable $G$ model. SNIa events may not be the good standard candles we take them for, unless a variation in $G$ is taken into account. Our models, A, and B, attempt to do just that, i.e., make reasonable predictions for $d_{L}^{\prime} / d_{L}$ given a specific redshift.

\section{Summary and Conclusion}

Cosmic inflation, i.e., the rapid expansion of the universe in its earliest phase, has been considered assuming a cosmologically varying $G$. The Friedmann equation, in particular, is invoked to argue that Hubble expansion is only possible if $G$ is unequal to zero. If $G$ varies with cosmological time, then it must be an intrinsic property of the vacuum. Moreover, it must be related to a scalar field, $\varphi$, as first suggested by Jordan. The Planck mass squared, $M_{P l}^{2}$, is no longer a constant, but related to $\varphi^{2}$, as shown explicitly in Equation (1-2). The $\varphi$ may also be interpreted as a new type of inflaton field, one associated with a time varying $G$, surfacing at a temperature we estimate to be about 10E22 Kelvin.

Two models for a time varying $G^{-1}$ were presented, models $\mathrm{A}$, and $\mathrm{B}$, given by Equations (1-4) and (1-6), respectively. Both functions rise dramatically at their respective inception temperatures, 6.20E21 Kelvin for model A, and 7.01E21 Kelvin for model B. In fact, at about these temperatures, both models give a $G^{-1}$ value, which is proportional to, $1 / T$, where $T$ is the CBR temperature. The cosmological scale parameter, “a”, is related to CBR temperature by way of the equation, $a=T_{0} / T=(1+z)^{-1}$. Eventually, as the universe cools upon expansion, $G^{-1}$ levels and tapers off and approaches a saturation value, a constant in the limit where, $a \rightarrow \infty$. 
There are only two coupling constants in nature, which have an inherent canonical dimension. The first is the Fermi constant, $G_{F}$, in the theory of weak interactions. We now know that, in reduced units, $G_{F}^{-1}$ is essentially equal to, $M_{W_{ \pm}}^{2}$, where, $M_{W_{ \pm}}$is the mass of the $W_{ \pm}$boson. This is at low energies. At high energies, above $100 \mathrm{GeV}$, the $G_{F}$ value increases. The second coupling constant with inherent canonical dimension is Newton's constant. In fact, it has exactly the same canonical dimension as, $G_{F}$. If we replace the mass of the $W_{ \pm}$ boson squared by the Planck mass squared, then we have an entirely analogous situation with regards to gravity. Keep in mind that the theory of weak interactions, in itself, is not renormalizable at high energies. It is only the broader electro-weak interaction, which is consistent at high energy/momentum transfers or exchanges. We believe that a similar situation holds for gravity. The Fermi constant, $G_{F}$, is definitely an order parameter. At very high temperatures, the mass of the $W_{ \pm}$boson becomes relatively unimportant, and we have, $G_{F}^{-1} \sim\left(p^{2}-M_{W_{ \pm}}^{2}\right)$ approaching zero. The, $p_{\mu}$, is the momentum, of the mediating boson. At low temperatures, $G_{F}^{-1} \sim M_{W_{ \pm}}^{2}$ reduces to a constant. We believe the same scenario holds true for gravity and Newton's constant, just at a much higher threshold temperature of approximately, 10E22 Kelvin.

Using our two models for, $G^{-1}(a)$, we predict very high values for the Hubble parameter, at inception of $G^{-1}$. The values, which were obtained, were given by equations, (1-12a, b), and (1-13a, b). We believe that these expansion rates could be interpreted as inflation, where the "inflaton" field of inflation is replaced by Jordan's scalar field, $\varphi$, above. Beyond this point, temperature-wise, $G$, simply did not exist, and there was no expansion of space, as determined by the Friedmann equation. It is hypothesized that when the universe started to expand, it had a finite size, of the order of the earth's radius. In the $\Lambda$ CDM model, expansion started from a singularity.

Finally, a variable $G$ model cannot be dismissed based on SNIa events. In fact, there is now some very solid observational/astronomical evidence indicating otherwise. A variable $G$ may be needed to interpret SNIa energy release properly, as well as rise and fall times for these events. See Equations (2-1) and (2-2). Specific predictions for luminosity distance are possible using our two models for, $G^{-1}(a)$, models A, and B. As specific examples, Equations (2-4) and (2-6), are results that apply for $z=1 / 2$. And for a larger redshift, $z=2 / 3$, we obtain Equations (2-7) and (2-8). It may well turn out that SNIa events, in order to serve as good standard candles, need to include a variation in Newton's constant, $G$. Without taking into account a weakening $G$ with an increase in cosmological time, erroneous results and interpretations occur. Dark energy may have more to do with a weakening in $G$ with decreased redshift, than anything else.

\section{Acknowledgements}

The author would like to thank his children, Kira, Juergen, and Henry for simply being who they are. 


\section{Conflicts of Interest}

The author declares no conflicts of interest regarding the publication of this paper.

\section{References}

[1] Pilot, C. (2019) Is Quintessence an Indication of a Time-Varying Gravitational Constant? Journal of High Energy Physics, Gravitation and Cosmology, 5, 41-81. https://doi.org/10.4236/jhepgc.2019.51003

[2] Pilot, C. (2019) The Age of the Universe Predicted by a Time-Varying G Model? Journal of High Energy Physics, Gravitation and Cosmology, 5, 928-934. https://doi.org/10.4236/jhepgc.2019.53048

[3] Dirac, P.A.M. (1937) The Cosmological Constants. Nature, 139, 323. https://doi.org/10.1038/139323a0

[4] Dirac, P.A.M. (1938) A New Basis for Cosmology. Proceedings of the Royal Society of London A, 165, 199-208. https://doi.org/10.1098/rspa.1938.0053

[5] Dirac, P.A.M. (1974) Cosmological Models and the Large Numbers Hypothesis. Proceedings of the Royal Society of London A, 338, 439-446. https://doi.org/10.1098/rspa.1974.0095

[6] Jordan, P. (1937) G Has to Be a Field. Naturwissenschaften, 25, 513-517. https://doi.org/10.1007/BF01498368

[7] Jordan, P. (1949) Formation of Stars and Development of the Universe. Nature, 164, 637-640. https://doi.org/10.1038/164637a0

[8] Haber, H. (1966) Die Expansion der Erde [The Expansion of the Earth]. In: Vieweg, F. and Sohn, B., Eds., Unser Blauer Planet [Our Blue Planet], Vol. 48, Vol. 52, Rororo Sachbuch [Rororo Nonfiction] (in German) Rororo Taschenbuch Ausgabe [Rororo Pocket Edition], Rowohlt Verlag, Reinbek, 54-55.

[9] Kragh, H. (2015) Pascual Jordan, Varying Gravity, and the Expanding Earth. Physics in Perspective, 17, 107-134. https://doi.org/10.1007/s00016-015-0157-9

[10] Wilkinson Microwave Anisotropy Probe (2013) WMAP Produces New Results: WMAP 9-Year Results Released. https://map.gsfc.nasa.gov/news

[11] Guth, A.H. (1981) Infiationary Universe: A Possible Solution to the Horizon and Flatness Problems. Physical Review D, 23, 347-356.

https://journals.aps.org/prd/pdf/10.1103/PhysRevD.23.347 https://doi.org/10.1103/PhysRevD.23.347

[12] Linde A.D. (1983) Chaotic Inflation. Physics Letters B, 129, 177-181. https://doi.org/10.1016/0370-2693(83)90837-7

[13] Linde A.D. (1994) Hybrid Inflation. Physical Review D, 49, 748-754. https://doi.org/10.1103/PhysRevD.49.748

[14] Kolb, E.W. and Turner, M.S. (1989) The Early Universe. Addison-Wesley, Boston.

[15] Baumann, D.D. (2015) Cosmology: Part III Mathematical Tripos. http://theory.uchicago.edu/ liantaow/my-teaching/dark-matter-472/lectures.pdf

[16] Mather, J.C., Fixsen, D.J., Shafer, R.A., Mosier, C. and Wilkinson, D.T. (1999) Calibrator Design for the COBE Far-Infrared Absolute Spectrophotometer (FIRAS). The Astrophysical Journal, 512, 511-520. https://doi.org/10.1086/306805

[17] Husdal, L. (2016) On Effective Degrees of Freedom in the Early Universe. Galaxies, 4, 78-113. https://doi.org/10.3390/galaxies4040078 
[18] Lemaitre, G. (1946) The Primeval Atom-An Essay on Cosmogony. In: Munitz, M.K., Ed., Theories of the Universe, Free Press, New York.

[19] Lemaitre, G. (1931) The Evolution of the Universe: Discussion. Nature, 128, 699-701.

[20] Lemaitre, G. (1927) Un Univers Homogène de Masse Constante et de Rayon Croissant Rendant Compte de la Vitesse Radiale des Nébuleuses Extragalactiques. Annals of the Scientific Society of Brussels, A47, 49-59.

[21] Lemaitre, G. (1931). The Beginning of the World from the Point of View of Quantum Theory. Nature, 127, 706. https://doi.org/10.1038/127706b0

[22] Bars, I. and Terning, J (2009) Extra Dimensions in Space and Time. Springer, Berlin. https://doi.org/10.1007/978-0-387-77638-5

[23] Lineweaver, C and Davis, T.M. (2005) Misconceptions of the Big Bang. Scientific American, 292, 24-33.

[24] Thorsett, S.E. (1996) The Gravitational Constant, the Chandrasekhar Limit, and Neutron Star Masses. Physical Review Letters, 77, 1432-1435. https://doi.org/10.1103/PhysRevLett.77.1432

[25] Gaztanaga, E., Garcia-Berro, E., Isern, J., Bravo, E. and Dominguez, I. (2001) Bounds on the Possible Evolution of the Gravitational Constant from Cosmological Type-Ia Supernovae. Physical Review D, 65, Article ID: 023506. https://doi.org/10.1103/PhysRevD.65.023506

[26] Garcia-Berro, E., Kubyshin, Y., Loren-Aguilar, P. and Isern, J. (2006) The Variation of the Gravitational Constant Inferred from the Hubble Diagram of Type Ia Supernovae. International Journal of Modern Physics D, 15, 1163-1174. https://doi.org/10.1142/S0218271806008772

[27] Garcia-Berro, J.I. and Kubyshin, Y.A. (2007), Astronomical Measurements and Constraints on the Variability of Fundamental Constants. The Astronomy and Astrophysics Review, 14, 113-170. https://doi.org/10.1007/s00159-006-0004-8 